\title{
Desempleo y salud mental en la población de origen hispano en Estados Unidos: un análisis epidemiológico
}

\author{
Unemployment and mental health among Hispanics in the US: \\ an epidemiological analysis
}

Maritza Caicedo ${ }^{1}$

Edwin van Gameren ${ }^{2}$
${ }^{1}$ Instituto de Investigaciones Sociales, Universidad Nacional Autónoma de México. Circuito Mario de la Cueva, Ciudad Universitaria. 04510 Ciudad de México DF México. caicedor@unam.mx ${ }^{2}$ Centro de Estudios Económicos, El Colegio de México. Ciudad de México DF México.

\begin{abstract}
The high unemployment rates observed in the United States during the recent economic crisis, that moreover vary between ethnic groups, make it relevant to inquire into how this problem is linked to the mental health of employed and unemployed persons. Therefore, in this paper the relationship between unemployment and mental health among Mexican immigrants, Mexicans born in the US, and other Hispanics in comparison with non-Hispanic native whites and Afro-Americans in the US is analyzed. To achieve this objective prevalence, prevalence ratios and odds ratios for the population between 18 and 65 years of age in the labor force was calculated using data from the 1999 and 2009 National Health Interview Surveys. It was seen that in times of crisis the prevalence of Non-Specific Psychological Disorders (NSPD) in the labor force increased in all ethnic groups. The prevalence ratios indicate that the unemployed face a higher risk of suffering from NSPD than the employed, especially for the non-Mexican Hispanics. Mexican immigrants, in contrast, show the lowest risks.
\end{abstract}

Key words Unemployment, Mental health, Hispanics, Prevalence, Odds ratio
Resumen Las altas tasas desempleo observadas en Estados Unidos durante la reciente crisis económica, que además son diferenciadas de acuerdo al origen étnico, hacen pertinente indagar acerca de cómo este problema se relaciona con la salud mental de los empleados y desempleados. Por tanto, en este artículo analizamos la relación entre desempleo y salud mental de los inmigrantes mexicanos, mexicanos nacidos en EE.UU, y otros hispanos en comparación con nativos blancos no hispanos y afroestadounidenses. Para alcanzar este propósito calculamos prevalencias, razones de prevalencias y razones de momios en la población entre 18 y 65 años en la fuerza laboral. Utilizamos información de la National Health Interview Survey (1999 y 2009). Encontramos que en tiempos de crisis aumenta la prevalencia de Tensión Psicológica no Específica (TPNE) en la fuerza laboral para todos los grupos étnicos. Las razones de prevalencias indican que los desempleados tienen mayor riesgo de presentar una TPNE que los empleados, particularmente los hispanos no mexicanos, en contraste, los inmigrantes mexicanos muestran riesgos más bajos.

Palabras clave Desempleo, Salud mental, Hispanos, Prevalencias, Razón de momios 


\section{Introducción}

No cabe duda que el aumento del desempleo es una de las consecuencias directas de las crisis económicas, más allá de reflejar el deterioro o el débil estado de una economía, afecta distintas dimensiones de la vida social y familiar e incluso la salud mental de quienes lo viven ${ }^{1-5}$. En Estados Unidos el desempleo ha estado correlacionado con factores como la raza, el lugar de origen, el sexo y la edad de los trabajadores ${ }^{6-8}$. De acuerdo con información censal, en 1970 la tasa de desempleo de la población total fue de 4.3\%, en 1980 y 1990 de $6.3 \%$ y en 2000 de $5.7 \%$. En los mismos años las tasas de desempleo de los inmigrantes latinoamericanos y caribeños superaron ostensiblemente estos valores $(5.9 \%, 8.6 \%, 10.3 \%$, y $8.9 \%$, respectivamente). Los afroestadounidenses registraron tasas todavía mayores: $6.9 \%, 11.6 \%$, $11.4 \%$ y $12.0 \%$, respectivamente .

En años recientes se ha constatado que en tiempos de crisis económica, las diferencias en las tasas de desempleo entre nativos blancos y estos grupos incrementan ${ }^{10}$. Por ejemplo, en 2006, la tasa de desempleo de los nativos blancos no hispanos fue $4.2 \%$, y la de los inmigrantes mexicanos de $4.8 \%$. En 2010, en plena crisis, la tasa para los primeros incrementó a $8.7 \%$, para los mexicanos de reciente arribo - con menos de 10 años en Estados Unidos - a 11.9\% y para los mexicanos con más de 10 años en el país a 12.8\%. En los años señalados los afroestadounidenses pasaron de una tasa de $9.9 \%$ a $17.1 \%{ }^{11}$.

Vale mencionar que desde los setenta la reestructuración de la economía y del mercado de trabajo estadounidense, en combinación con el bajo nivel de escolaridad y ocupacional de algunos trabajadores y la discriminación laboral que muchos experimentan, han favorecido el aumento del desempleo entre algunos grupos étnicos ${ }^{12}$.

En este artículo, desde un enfoque epidemiológico, analizamos la relación entre desempleo y Tensiones Psicológicas No Específicas - depresión y ansiedad -, en varios grupos étnicos nativos y población de origen hispano - en los Estados Unidos. Comparamos los años 1999 una situación económica favorable - y 2009, un año de crisis. Partimos de la hipótesis que la Tensión Psicológica No Específica (TPNE) aumenta en la fuerza laboral de los Estados Unidos en tiempos de crisis y el riesgo de experimentarla es mayor en los desempleados, pero hay diferencias de acuerdo al grupo étnico. La teoría sociológica encuentra en la estratificación social una de las explicaciones a los problemas o enfermedades mentales, de acuerdo con ésta, las personas de más bajos recursos tienden a presentar menor salud mental que las que poseen mayores recursos y estatus socioeconómico ${ }^{5}$. Apoyados en esta idea, planteamos que los desempleados pertenecientes a los grupos étnicos en mayor vulnerabilidad socioeconómica podrían tener mayor riesgo de experimentar una TPNE. Esperamos que la población de origen hispano y afroestadounidenses tengan riesgos más altos. En los primeros por experimentar mayores vulnerabilidades intrínsecas a la migración y en los segundos por la histórica desventaja social que han vivido en Estados Unidos. Estos factores sumados a la condición de desempleado pueden hacerlos más proclives a presentar una TPNE, mientras que para los nativos blancos no hispanos, por su mejor situación socioeconómica, el riesgo podría ser menor?.

\section{Antecedentes}

\section{El concepto de salud mental}

De acuerdo con la OMS la salud mental no es sólo la ausencia de trastornos mentales, se trata de "un estado de bienestar en el cual el individuo es consciente de sus propias capacidades, puede afrontar las tensiones normales de la vida, trabajar de forma productiva y contribuir a su comunidad"13. De acuerdo con Álvaro ${ }^{14}$, el concepto contiene una carga valorativa, pues las evaluaciones de los síntomas y procesos afectivos, cognitivos y comportamentales empleados para determinar si un individuo es sano o enfermo varían de acuerdo a las representaciones sociales y paradigmas científicos prevalecientes en cada contexto sociocultural y periodo histórico. Además, para el autor, aunque el concepto es la expresión de aspectos emocionales, cognitivos y comportamentales, constituye a la vez una realidad simbólica que se construye cultural e históricamente.

\section{Migración y salud mental}

Diversos estudios, desde distintas disciplinas, subrayan la importancia de observar los efectos de la migración en la salud mental de los inmigrantes. Bhugra y Jones ${ }^{15}$ señalan que el proceso migratorio en sí mismo y la subsecuente adaptación cultural y social de los inmigrantes a la sociedad receptora juegan un rol central en su salud mental. Vega et al. ${ }^{16}$ agregan que las características sociodemográficas de los inmigrantes, 
como la baja escolaridad, los bajos ingresos, el desempleo, las rupturas conyugales, así como el tiempo de permanencia en el país de destino están estrechamente relacionados con altas tasas de depresión entre inmigrantes.

Vega y Rumbaut ${ }^{17}$ señalan que la cohesión familiar y los valores tradicionales de las familias hispanas reducen la presencia de enfermedades mentales. Se señaló que la cultura mexicana ayuda a amortiguar factores de estrés en los individuos y que a la vez es más tolerante con los comportamientos anómalos que pudieran presentar miembros de la familia. Investigaciones posteriores dejaron claro que las limitaciones que poseen muchos hispanos con el idioma inglés limita el reporte y la búsqueda de atención profesional ante problemas de salud mental ${ }^{18-21}$.

Pesquisas etnográficas encontraron que la población de origen mexicano presenta enfermedades psiquiátricas en niveles similares a los observados entre los nativos blancos no hispanos ${ }^{22}$. Mientras que Alegría et al. ${ }^{23}$ han encontrado menor prevalencia de desordenes psiquiátricos entre inmigrantes de origen latinoamericano en comparación con la población de origen latinoamericano nacida en Estados Unidos y los nativos no hispanos.

Grant et al. ${ }^{24}$ señalaron que las bajas tasas de enfermedad mental entre inmigrantes mexicanos en relación con mexicoamericanos y nativos blancos no-hispanos obedecen a un efecto de selección de la migración. Es decir, los individuos con buena salud mental emigran más a Estados Unidos que aquellos que no gozan de esta condición, por lo tanto, el riesgo de padecer alguna enfermedad mental es menor para ellos. No hay consenso en este aspecto ${ }^{20}$.

\section{Desempleo y salud mental}

Existe un cúmulo de investigaciones desarrolladas en la década de los treinta que constataron la relación negativa entre desempleo y salud mental $^{25,26}$. En los años setenta los países desarrollados experimentaron una crisis económica que generó un crecimiento inusitado del desempleo. Esto dio lugar al desarrollo de investigaciones que comprobaron daño psicológico que genera el desempleo en los individuos ${ }^{27,28}$. Estudios recientes desde disciplinas como la psicología, psiquiatría, sociología, economía y demografía siguen dando cuenta de dicha relación ${ }^{29-38}$.

Garrido $^{39}$ puntualiza que las investigaciones actuales llevan a la permanente constatación que las personas desempleadas presentan menores niveles de bienestar psicológico, mayor grado de sentimientos depresivos y ansiedad, así como menor autoestima y satisfacción con la vida. Del Pozo et al. ${ }^{40}$ argumentan que cuando se pierde el empleo ocurre un rápido deterioro de la salud mental por la ausencia de "aportes psicosociales" como la solvencia económica, la seguridad física, la pérdida de habilidades laborales, la imposibilidad de predecir y planificar el futuro y la pérdida de una posición social valorada. Los autores muestran que con la prolongación del desempleo aumenta el deterioro de la salud mental.

Otros plantean que no existe una relación directa entre desempleo y salud mental. Schaufeli ${ }^{41}$ demuestra que se trata de una relación mediada por características personales como un alto nivel de autoestima y bajo nivel de inestabilidad mental, aspectos que protegen al individuo del estrés que puede producir el desempleo. También influyen factores como el soporte social, la actitud hacia el hecho de no trabajar y el compromiso que establece cada individuo con el trabajo, además de otros factores como el sexo, edad, nivel de ingresos del desempleado, satisfacción con trabajos previos, expectativas de retornar a un nuevo empleo, etc. ${ }^{41,42}$.

Ezzy $^{42}$ cuestiona los modelos teóricos desarrollados para explicar la relación entre desempleo y salud mental, porque éstos se centran en el individuo y frecuentemente excluyen los factores sociales e institucionales que contribuyen a la explicación del fenómeno. Agrega que la presión, la pérdida del sentido de sí mismo, las emociones "disfóricas" y las estrategias depresivas, se presentan solamente cuando una persona sufrió la pérdida de su rol social y no encuentra un rol alternativo a través del cual cumplir sus metas "autodefinitorias". Ello sugiere la necesidad de profundizar en los aspectos socioculturales que inciden en la relación desempleo-salud mental.

En síntesis, la relación entre desempleo y salud mental está mediada por un sinnúmero de factores psíquicos, demográficos, familiares, económicos, culturales y ambientales. No existe un enfoque único biológico, psicológico o sociológico que explique completamente los orígenes de tensiones psicológicas como la depresión y la ansiedad $^{43}$. El aporte de la sociología radica en mostrar cómo eventos especiales en la vida de un ser humano, las condiciones sociales, los sistemas culturales y los roles sociales afectan el estado mental de los individuos ${ }^{5}$. 


\section{La relación entre migración, desempleo y salud mental}

La migración complejiza la relación entre desempleo y salud mental. Los cambios que impone la migración desde el punto de vista social, económico, profesional, cultural muchas veces constituyen un foco de tensión en los inmigrantes; pero la migración, per se, no es responsable de la aparición de tensiones psicológicas, solo se convierte en factor de riesgo cuando se trata de población en alto grado de vulnerabilidad socioeconómica ${ }^{44}$.

Es claro que el contexto de recepción - las políticas migratorias del gobierno, las condiciones de inserción en el mercado de trabajo y las características de la comunidad étnica en que se insertan los inmigrantes - puede mitigar o potencializar la parición de tensiones psicológicas en los inmigrantes ${ }^{45}$. El contexto de recepción estadounidense para muchos inmigrantes de América Latina y el Caribe es adverso. Existe abundante bibliografía sobre la relación entre experiencias de discriminación en minorías étnicas $y$ el efecto negativo en su salud mental ${ }^{46-49}$, particularmente en el campo laboral ${ }^{50}$. Investigaciones recientes han evidenciado una asociación fuerte entre pobreza, ausencia de trabajo asalariado y angustia psicológica en inmigrantes en los lugares de inmigración ${ }^{51,52}$.

La desigualdad socioeconómica que muchas veces afrontan los inmigrantes es una de las explicaciones más socorridas para entender la presencia de tensiones psicológicas en ciertos individuos. Por ejemplo, las diferencias entre nativos e inmigrantes latinoamericanos en el acceso al empleo, a buenos salarios, y a la salud son indicadores de esa desigualdad, que entre otros aspectos, puede deberse al bajo capital humano y a la condición de indocumentados. Esta condición limita el ejercicio pleno de sus derechos ${ }^{9}$. En términos generales, hay una conjunción de factores sociales e individuales que en muchos casos ponen a los inmigrantes en gran desventaja socioeconómica.

\section{Metodología}

\section{Fuente de datos}

Para el análisis empleamos datos de la National Health Interview Survey (NHIS) de los años
1999 y 2009. Anualmente recolecta información sobre la salud de la población civil no institucionalizada de Estados Unidos. Se encuestan aproximadamente 35,000 hogares que contienen 85,000 personas. En cada hogar se selecciona a un adulto (de 18 años y más) a quien se aplica un cuestionario para recabar información general sobre el estado de salud, uso de servicios y conductas con respecto a la salud. Es representativa a nivel nacional, aunque la población afroestadounidense e hispana se encuentra sobrerrepresentada en la muestra; los ponderadores (disponibles en las bases de datos) compensan esto.

La fuente no permite distinguir entre inmigrantes documentados e indocumentados, lo cual hace una diferencia cualitativa en términos de integración socioeconómica a la sociedad receptora. Además, los bajos tamaños de muestra no nos permiten hacer un análisis para cada país de origen latinoamericano y caribeño, y la ausencia de variables sobre antecedentes de TPNE o de la duración del desempleo limitan un análisis más acucioso sobre la relación entre desempleo y salud mental. Para mayor información sobre aspectos metodológicos de la encuesta, véase el sitio de la NHIS ${ }^{53,54}$.

Seleccionamos los años mencionados por tratarse de momentos de relativa estabilidad económica (1999) y crisis económica (2009); en 1999 la tasa de desempleo en el país no superó el 4.0\% mientras que en 2009 se ubicó muy por encima de este valor y constituye la más alta de los últimos 20 años ${ }^{55}$.

Los análisis están dirigidos a las comparaciones entre grupos étnicos: los inmigrantes mexicanos; los mexicoamericanos, que son las personas nacidas en Estados Unidos que se autodefinieron como mexicanos; otros hispanos, que hace referencia a la población de origen hispano que pudo o no haber nacido en Estados Unidos - se incluye a toda la población latinoamericana y a los puertorriqueños y se excluye a la población de origen español -;los afroestadounidenses nacidos en EE.UU.; y la población nativa blanca no hispana. Se incluye sólo a la población en la fuerza laboral - empleados y desempleados - entre 18 y 65 años. Los desempleados son las personas que durante una semana previa a la aplicación de la encuesta no tuvieron un trabajo remunerado, durante las cuatro semanas anteriores a la aplicación de la encuesta estuvieron buscando activamente un empleo y contaban con disponibilidad de empezar a trabajar en cualquier momento $0^{55}$. 


\section{Escala de Tensión Psicológica No Específica}

Empleamos el concepto de Tensión Psicológica No Específica (TPNE) acuñado por Kessler y Mroczek $^{56}$. Se trata de un concepto operativo que permite establecer el riesgo de presentar depresión o ansiedad a través de la construcción de una escala, pero no es posible precisar si se trata de una u otra. La NHIS incluye las seis preguntas de la escala abreviada de Kessler et al. ${ }^{57}$. A una muestra de adultos de 18 años y más se les preguntó lo siguiente: Con qué frecuencia durante los últimos 30 días usted se sintió... ¿Tan triste que nada le alegraba?, ¿Nervioso?, ¿Desesperado?, ¿Intranquilo o inquieto?, ¿Que todo era un esfuerzo? y ¿Sin mérito alguno? Las respuestas posibles a estas interrogantes son: (0) nunca, (1) muy pocas veces, (2) parte del tiempo, (3) casi todo el tiempo, y (4) todo el tiempo. Al sumar los puntajes (0-4) asignados a las respuestas sobre las seis preguntas se construye una escala de medición del riesgo de experimentar una TPNE; la escala se mueve entre 0 y $24^{58}$.

\section{Análisis estadístico}

En nuestros datos de corte transversal calculamos las prevalencias y realizamos Pruebas t para cada grupo étnico de la diferencia entre las prevalencias de desempleo y de TPNE en 1999 y 2009, y Pruebas F para cada año de la diferencia entre las prevalencias de TPNE y desempleo en las submuestras étnicas. Calculamos medidas de asociación - razones de prevalencia y razones de momios de prevalencia -, según año y grupo étnico. Ambos indicadores miden el grado de asociación entre una "enfermedad" y determinada exposición. La razón de prevalencia nos permite establecer cuántas veces es más probable que un desempleado presente una TPNE en comparación con un empleado. Cuando la razón de prevalencia es igual a uno, se debe entender que la prevalencia de TPNE es igual en empleados y en desempleados. Un valor superior a uno indica mayor prevalencia de TPNE en desempleados e inferior a uno mayor prevalencia en empleados. Las razones de momios de prevalencia nos dan cuenta del exceso que tienen los desempleados de presentar una TPNE frente a no presentarla en comparación al exceso de los empleados de presentar una TPNE frente a no presentarla ${ }^{59}$. Su interpretación es similar; el valor uno indica que la posibilidad de tener una TPNE es igual entre empleados y desempleados, por encima de uno la posibilidad de presentar una TPNE es mayor en desempleados y menor a uno indica que es mayor en empleados. [falta a citação 60]

\section{Resultados}

Entre 1999 y 2009 hubo un aumento importante en el nivel de desempleo en todos los grupos. En el primer año la tasa de desempleo de los nativos blancos no hispanos fue de $2.1 \%$ y en el segundo incrementó a $7.8 \%$ (Tabla 1).

Tabla 1. Tasas de desempleo en la población entre 18 y 65 años en la fuerza laboral, según grupo étnico, Estados Unidos, 1999 y 2009.

\begin{tabular}{|c|c|c|c|c|c|c|c|}
\hline \multirow[b]{2}{*}{ Grupo étnico } & \multicolumn{3}{|c|}{1999} & \multicolumn{3}{|c|}{2009} & \multirow[b]{2}{*}{$\begin{array}{c}\text { Prueba } \\
\mathbf{t}^{\mathrm{b}}\end{array}$} \\
\hline & $\mathbf{n}$ & $\mathbf{N}$ & $\begin{array}{c}\text { Tasa de } \\
\text { desempleo }^{\mathrm{d}}\end{array}$ & $\mathbf{n}$ & $\mathbf{N}$ & $\begin{array}{c}\text { Tasa de } \\
\text { desempleo }^{\mathrm{d}}\end{array}$ & \\
\hline Nativos blancos no hispanos & 12038 & $92,207,677$ & 2.1 & 9276 & $94,630,102$ & 7.8 & 0.0000 \\
\hline Afroestadounidenses & 2374 & $13,140,345$ & 5.6 & 2363 & $15,163,110$ & 15.0 & 0.0000 \\
\hline Mexicoamericanos & 913 & $3,905,359$ & 4.2 & 909 & $6,017,574$ & 11.9 & 0.0000 \\
\hline Inmigrantes mexicanos & 976 & $4,424,236$ & 3.1 & 1224 & $7,841,429$ & 11.3 & 0.0000 \\
\hline Otros hispanos ${ }^{\mathrm{a}}$ & 1059 & $4,718,101$ & 2.9 & 1173 & $7,105,348$ & 13.4 & 0.0000 \\
\hline Prueba F (todos) ${ }^{\mathrm{c}}$ & & & 0.0000 & & & 0.0000 & \\
\hline Prueba F (sin blancos) ${ }^{c}$ & & & 0.0005 & & & 0.0103 & \\
\hline
\end{tabular}

Fuente: Cálculos propios con base en NHIS, 1999 y 2009; datos ponderados. ${ }^{a}$ Se incluye a los puertorriqueños. ${ }^{\mathrm{b}}$ p-valores de Pruebas t; comprueban que, en cada grupo étnico, la diferencia entre las tasas de desempleo en los dos años observados es estadísticamente significativa. ' $\mathrm{p}$-valores de Pruebas F; comprueban que las tasas de desempleo en 1999 y 2009, respectivamente, no son iguales en todos los grupos étnicos. ${ }^{\mathrm{d}}$ La NHIS no está especializada en recabar información laboral, por tanto, existen algunas diferencias con la información obtenida de la fuente especializada en información laboral ${ }^{60}$. 
Tabla 2. Prevalencias de Tensión Psicológica No Específica (escala Kessler-6) en la población entre 18 y 65 años en la fuerza laboral, según año y grupo étnico, Estados Unidos, 1999 y 2009.

\begin{tabular}{|c|c|c|c|}
\hline Grupo étnico & 1999 & 2009 & $\begin{array}{c}\text { Prueba } \\
\mathbf{t}^{\mathrm{b}}\end{array}$ \\
\hline \multicolumn{4}{|c|}{ Panel A: Alguna TPNE (1-24) } \\
\hline Nativos blancos no hispanos & 48.7 & 57.0 & 0.0000 \\
\hline Afroestadounidenses & 38.6 & 53.7 & 0.0000 \\
\hline Mexicoamericanos & 47.6 & 53.5 & 0.0149 \\
\hline Inmigrantes mexicanos & 32.0 & 45.3 & 0.0000 \\
\hline Otros hispanos ${ }^{\mathrm{a}}$ & 37.6 & 48.4 & 0.0000 \\
\hline Prueba F (todos) $)^{\mathrm{b}}$ & 0.0000 & 0.0000 & \\
\hline Prueba F (sin blancos) $)^{\mathrm{b}}$ & 0.0000 & 0.0000 & \\
\hline \multicolumn{4}{|c|}{ Panel B: TPNE leve o severa $(7-24)^{\mathrm{d}}$} \\
\hline Nativos blancos no hispanos & 7.9 & 9.3 & 0.0002 \\
\hline Afroestadounidenses & 8.9 & 12.6 & 0.0000 \\
\hline Mexicoamericanos & 9.4 & 7.9 & 0.2238 \\
\hline Inmigrantes mexicanos & 5.0 & 8.6 & 0.0018 \\
\hline Otros hispanos ${ }^{\mathrm{a}}$ & 6.9 & 11.2 & 0.0008 \\
\hline Prueba F (todos) ${ }^{\mathrm{b}}$ & 0.0136 & 0.0001 & \\
\hline Prueba F (sin blancos) $)^{\mathrm{b}}$ & 0.0006 & 0.0000 & \\
\hline \multicolumn{4}{|c|}{ Panel C: TPNE severa $(13-24)^{\mathrm{d}}$} \\
\hline Nativos blancos no hispanos & 1.4 & 2.0 & 0.0006 \\
\hline Afroestadounidenses & 2.2 & 2.9 & 0.1470 \\
\hline Mexicoamericanos & 2.4 & 1.4 & 0.1289 \\
\hline Inmigrantes mexicanos & 1.2 & 1.0 & 0.6480 \\
\hline Otros hispanos ${ }^{\mathrm{a}}$ & 2.0 & 2.9 & 0.1669 \\
\hline Prueba F (todos) ${ }^{c}$ & 0.0078 & 0.0024 & \\
\hline Prueba F (sin blancos) ${ }^{c}$ & 0.2554 & 0.0003 & \\
\hline
\end{tabular}

Fuente: Cálculos propios con base en NHIS, 1999 y 2009; datos ponderados. ${ }^{\mathrm{a} S e}$ incluye a los puertorriqueños. ${ }^{\mathrm{b}} \mathrm{p}$-valores de Pruebas t; indican que las diferencias en las prevalencias entre 1999 y 2009 para TPNE severa solo son estadísticamente significativas para los nativos blancos. ${ }^{c} \mathrm{p}$-valores de Pruebas F; comprueban que las diferencias en las prevalencias de TPNE entre los grupos étnicos observados son estadísticamente significativas (con la excepción de la prueba sin incluir a los nativos blancos no hispanos en el año 1999). ${ }^{\mathrm{d}} \mathrm{El}$ rango del panel de TPNE es acumulativo.

Las tasas de desempleo de los afroestadounidenses duplican las de los nativos blancos no hispanos en los dos años. En 1999 tuvieron una tasa de $5.6 \%$ y en 2009 subió a $15.0 \%$. Como era de esperarse, el desempleo en este periodo de tiempo afectó severamente a los inmigrantes. Los mexicanos pasaron de una tasa de 3.1\% en 1999 a $11.3 \%$ en 2009 . En 1999 la tasa de los otros hispanos fue $2.9 \%$ y en 2009 ascendió a 13.4\%. En los años mencionados, los mexicoamericanos pasaron de una tasa de 4.2 a $11.9 \%$.
En la Tabla 2 se presentan prevalencias de TPNE en la fuerza laboral en tres niveles: Panel A, personas con alguna TPNE - no se establece el nivel, solamente si tiene una TPNE -; panel B, personas con una TPNE leve o severa y en el C personas con una TPNE severa. Se habla de una TPNE severa cuando el índice se ubica por encima de los 12 puntos $^{58}$; valores entre 1-6 y 7-12 se consideran como una TPNE muy leve y leve, respectivamente. En el panel A se observa un incremento en la prevalencia de alguna TPNE en todos los grupos entre 1999 y 2009. En el primer año todos los valores se ubicaron por debajo del $50 \%$, destacando la situación de los inmigrantes mexicanos cuya prevalencia de alguna TPNE estuvo muy por debajo de los valores observados en los demás grupos (32.0\%). En el segundo año la prevalencia para los nativos blancos no hispanos fue $57.0 \%$ y para los afroestadounidenses $53.7 \%$, mientras que los inmigrantes mexicanos y los otros hispanos estuvieron por debajo de este valor, siendo los inmigrantes mexicanos los que presentaron el más bajo (45.3\%). El panel B indica que en 2009 también incrementó la cantidad de personas con una TPNE leve o severa, excepto en el caso de los mexicoamericanos, siendo los afroestadounidenses y otros hispanos quienes presentaron los valores más altos (12.6 y $11.2 \%$, respectivamente). En el panel $\mathrm{C}$, los inmigrantes mexicanos presentaron el valor más bajo, y no hubo aumentos en TPNE severa en los mexicanos (nacidos en EE.UU. e inmigrantes), mientras que en los demás grupos se observan incrementos en este nivel de TPNE. Las pruebas F y t revelaron que tanto las diferencias en las tasas de desempleo como en las prevalencias de TPNE entre los grupos observados en los dos años son estadísticamente significativas (Tablas 1 y 2).

Aunque no contamos con elementos de peso para afirmar que la crisis económica tiene un efecto negativo en la salud mental de la población, el fuerte crecimiento del desempleo y el aumento de personas con una TPNE en los años observados, nos sugiere una posible relación entre los dos fenómenos. Para un análisis de la misma, observamos prevalencias y riesgos en la fuerza laboral.

En la Tabla 3 se presentan medidas de frecuencia y asociación de TPNE para empleados y desempleados, según grupo étnico y año. En 1999 la prevalencia de alguna TPNE entre desempleados se ubicó por encima del 52\% en todos los grupos, excepto en los inmigrantes mexicanos (34.2\%). El mayor valor se registró entre los mexicoamericanos (66.3\%). En el caso 
Tabla 3. Medidas de frecuencia y efecto de Tensión Psicológica No Específica en población entre 18 y 65 años en la fuerza laboral, según año y grupo étnico, Estados Unidos, 1999 y 2009.

\begin{tabular}{|c|c|c|c|c|c|}
\hline Grupo étnico & $\begin{array}{c}\text { Prevalencia en } \\
\text { desempleados } \\
\text { (1) }\end{array}$ & $\begin{array}{c}\text { Error } \\
\text { Estándar } \\
\quad(2)\end{array}$ & $\begin{array}{c}\text { Prevalencia } \\
\text { en empleados } \\
(3)\end{array}$ & $\begin{array}{c}\text { Error } \\
\text { Estándar } \\
\quad(4)\end{array}$ & $\begin{array}{c}\text { Razón de } \\
\text { prevalencia }^{\mathrm{a}} \\
\text { (5) }\end{array}$ \\
\hline & & & 1999 & & \\
\hline Nativos blancos no hispanos & 0.535 & 0.031 & 0.486 & 0.005 & 1.100 \\
\hline Afroestadounidenses & 0.521 & 0.045 & 0.378 & 0.010 & 1.378 \\
\hline Mexicoamericanos & 0.663 & 0.076 & 0.468 & 0.017 & 1.416 \\
\hline Inmigrantes mexicanos & 0.342 & 0.083 & 0.320 & 0.015 & 1.070 \\
\hline \multirow[t]{2}{*}{ Otros hispanos ${ }^{\mathrm{b}}$} & 0.529 & 0.079 & 0.371 & 0.015 & 1.423 \\
\hline & & & 2009 & & \\
\hline Nativos blancos no hispanos & 0.752 & 0.016 & 0.555 & 0.005 & 1.354 \\
\hline Afroestadounidenses & 0.654 & 0.025 & 0.516 & 0.011 & 1.267 \\
\hline Mexicoamericanos & 0.645 & 0.046 & 0.520 & 0.018 & 1.241 \\
\hline Inmigrantes mexicanos & 0.561 & 0.042 & 0.439 & 0.015 & 1.280 \\
\hline Otros hispanos ${ }^{\mathrm{b}}$ & 0.674 & 0.039 & 0.455 & 0.016 & 1.483 \\
\hline \multirow[t]{2}{*}{ Grupo étnico } & \multicolumn{2}{|c|}{$\begin{array}{c}\text { Intervalo de confianza } \\
\text { razón de prevalencia } \\
(6)\end{array}$} & $\begin{array}{c}\text { Razón de momios } \\
\text { de prevalencia }{ }^{\mathrm{a}} \\
\text { (7) }\end{array}$ & \multicolumn{2}{|c|}{$\begin{array}{c}\text { Intervalo de confianza } \\
\text { del RMP } \\
(8)\end{array}$} \\
\hline & \multicolumn{5}{|c|}{1999} \\
\hline Nativos blancos no hispanos & \multicolumn{2}{|c|}{$0.978-1.236$} & 1.214 & \multicolumn{2}{|c|}{$0.945-1.560$} \\
\hline Afroestadounidenses & \multicolumn{2}{|c|}{$1.161-1.635$} & 1.788 & \multicolumn{2}{|c|}{$1.259-2.539$} \\
\hline Mexicoamericanos & \multicolumn{2}{|c|}{$1.118-1.794$} & 2.233 & \multicolumn{2}{|c|}{$1.129-4.419$} \\
\hline Inmigrantes mexicanos & \multicolumn{2}{|c|}{$0.646-1.772$} & 1.107 & \multicolumn{2}{|c|}{$0.515-2.379$} \\
\hline \multirow[t]{2}{*}{ Otros hispanos ${ }^{\mathrm{b}}$} & \multicolumn{2}{|c|}{$1.009-2.008$} & 1.898 & \multicolumn{2}{|c|}{$0.922-3.906$} \\
\hline & & 2009 & & \\
\hline Nativos blancos no hispanos & \multicolumn{2}{|c|}{$1.294-1.418$} & 2.428 & \multicolumn{2}{|c|}{$2.040-2.889$} \\
\hline Afroestadounidenses & \multicolumn{2}{|c|}{$1.162-1.382$} & 1.772 & \multicolumn{2}{|c|}{$1.399-2.243$} \\
\hline Mexicoamericanos & \multicolumn{2}{|c|}{$1.063-1.449$} & 1.678 & \\
\hline Inmigrantes mexicanos & \multicolumn{2}{|c|}{$1.089-1.504$} & 1.637 & \multicolumn{2}{|c|}{$\begin{array}{l}1.106-2.54 \\
1.147-2.338\end{array}$} \\
\hline Otros hispanos ${ }^{\mathrm{b}}$ & \multicolumn{2}{|c|}{$1.305-1.686$} & 2.484 & \multicolumn{2}{|c|}{$\begin{array}{l}1.147-2.338 \\
1740-3547\end{array}$} \\
\hline
\end{tabular}

Fuente: Cálculos propios con base en NHIS, 1999 y 2009; datos ponderados. Los intervalos de confianza son de $95 \%$.

${ }^{a}$ Comparación entre desempleados y empleados. ${ }^{\mathrm{b}}$ Se incluye a los puertorriqueños.

de la población empleada las prevalencias son significativamente menores en todos los grupos, excepto en los inmigrantes mexicanos, y ningún grupo registró valores superiores al 50\%. Los inmigrantes mexicanos presentaron la prevalencia más baja $(32.0 \%)$.

En 2009 hay aumentos en dichas prevalencias en todos los grupos, excepto en los mexicoamericanos. Entre los desempleados, los nativos blancos no hispanos registran el valor más alto $(75.2 \%)$, seguidos de los de otro origen hispano (67.4\%) y afroestadounidenses (65.4\%), mientras que los inmigrantes mexicanos presentaron la prevalencia más baja $(56.1 \%)$, pero muy superior a la observada en 1999. Se registra también un aumento de las prevalencias de TPNE entre la población empleada de todos los grupos, pero siguen siendo inferiores a las observadas entre la población desempleada.

Las razones de prevalencia nos indican que en 1999 los desempleados con mayor riesgo de presentar una TPNE se ubicaron entre los "otros hispanos" y los mexicoamericanos, donde un desempleado tiene 1.42 veces mayor riesgo de experimentar este tipo de síntomas que un individuo empleado del mismo grupo y le siguen los afroestadounidenses (1.38). Los intervalos de confianza indican que, en estos grupos, los desempleados tienen un riesgo significativamente mayor al de los empleados de tener una TPNE. 
Además, estos valores están por arriba del intervalo de confianza (de 95\%) de los nativos blancos, y de igual manera el valor calculado para los nativos blancos (1.10) se encuentra por debajo de los intervalos de confianza de los tres grupos, lo que sugiere que las diferencias entre nativos blancos y los otros tres grupos son reales.

En el año 2009 se observan cambios importantes. Los otros hispanos desempleados siguen presentando el mayor riesgo de experimentar una TPNE (1.48), en relación con la población del mismo grupo empleada. En este año la razón de prevalencia para los nativos blancos no hispanos fue 1.35, mientras que los afroestadounidenses, los inmigrantes mexicanos y los mexicoamericanos revelaron valores cercanos al 1.25, valores que se encuentran por debajo del los intervalos de confianza de los nativos blancos no hispanos y de los otros hispanos. Esto muestra un fuerte contraste con el año 1999. Además, los intervalos de confianza para 2009 indican que las diferencias entre empleados y desempleados son significativas para cada grupo étnico.

Las razones de momios de prevalencia confirman la tendencia observada. En 1999 los mexicoamericanos desempleados tuvieron el mayor riesgo de presentar una TPNE; 2.23 veces más probable que para un empleado. El segundo valor más alto lo presentaron los otros hispanos (1.90) y en tercer lugar se ubicaron los afroestadounidenses (1.79), los tres grupos con razones de momios de prevalencia por arriba del intervalo de confianza de los nativos blancos no hispanos. $\mathrm{Al}$ observar las razones de momios de prevalencia en 2009, encontramos que los afroestadounidenses y los mexicoamericanos pasan de un valor más alto a un valor por debajo del intervalo de confianza de los nativos blancos no hispanos.

Al controlar por sexo, edad y escolaridad encontramos que no hay cambios grandes en la composición de los grupos entre 1999 en 2009, y que los pequeños cambios observados ocurren en cada grupo étnico casi sin diferencias significativas. También se elaboraron análisis de acuerdo al sexo, la edad, y la escolaridad (que por razones de espacio no se incluyen en este artículo). El análisis por separado para hombres y mujeres confirmó los resultados de la Tabla 3. Los análisis por rangos decenales de edad y por nivel de educación sugieren que en general se mantiene la tendencia observada en la población completa; en particular, las razones de prevalencias y momios son más altos para los desempleados. Sin embargo, debido a que las muestras son más pequeñas se reduce la significancia.

\section{Discusión}

En síntesis, observamos que entre 1999 y 2009, el año más fuerte de la crisis financiera estadounidense, se produjo un aumento fuerte en las tasas de desempleo en cada grupo étnico. En el mismo periodo se registró un aumento de las prevalencias de presentar alguna TPNE, con diferencias importantes por grupo étnico - confirmándose la primera parte de nuestra hipótesis -, los incrementos de personas con una TPNE leve o severa y severa fueron bajos e incluso decrecientes en la población de origen mexicano. Por tanto, para los de origen mexicano hay un incremento relativamente fuerte en TPNE ligeras sin expansión a TPNE más fuertes.

Al comparar las razones de prevalencia y los riesgos de presentar una TPNE entre desempleados y empleados, encontramos que existe una relación estrecha entre desempleo y el riesgo de presentar una TPNE. El riesgo es mayor para las personas que se encuentran desempleadas, pero existen diferencias de acuerdo al grupo étnico que no son constantes en el tiempo. Los resultados confirman parte de la hipótesis; sin embargo, los grupos más vulnerables, en términos socioeconómicos, - los inmigrantes mexicanos no fueron quienes presentaron el mayor riesgo de experimentar una TPNE.

Llama poderosamente la atención la baja prevalencia de una TPNE en los inmigrantes mexicanos, en relación con los demás grupos, y particularmente en relación con los demás hispanos, así como también que sea el grupo que presenta las razones de prevalencia y las razones de momios de prevalencia más bajas, lo que podría sugerir que su salud mental es menos afectada por el desempleo. Este resultado pareciera comprobar la "paradoja hispana"18,23,24,61-63.

Varios factores podrían influir en los resultados encontrados. Es posible que la menor prevalencia de TPNE entre inmigrantes se asocie a la selección que se genera de las personas más sanas, jóvenes y con capacidad de trabajar. La mayor tolerancia frente a la enfermedad mental y el soporte de redes de apoyo familiares y de amigos ante la adversidad es otro aspecto que podría explicar la baja propensión de los inmigrantes mexicanos a experimentar este tipo de síntomas ${ }^{64}$. Asimismo, las diferencias culturales podrían incidir en la actitud de los individuos frente al hecho de aceptar estados de ánimo negativos.

Además se deben contemplar las condiciones de vida que tuvieron en el lugar de origen y las expectativas de vida en el contexto de recep- 
ción. Es decir, alguien que partió huyendo de la pobreza y el desempleo en el contexto de origen, es posible que pueda ajustarse a condiciones mínimas de vida en las sociedades de llegada, que en muchos casos podrían ser mejores a las que tenía en su contexto de origen. Ello sin duda podría contrarrestar sentimientos de frustración y fracaso generados por el desempleo y/o las precarias condiciones laborales. También se debe tener presente que los inmigrantes tienen menores periodos de duración en el desempleo que la población nativa blanca no hispana que podrían reducir el riesgo de presentar una TPNE ${ }^{65}$.

Es posible que la connotación negativa de las preguntas Kessler-6, genere, particularmente en el caso de los inmigrantes mexicanos, el no reconocimiento de síntomas depresivos o de ansiedad. En general, dado que contamos con información de tipo transversal y no controlamos los cambios en la composición de los grupos observados - particularmente el stock de inmigrantes está sujeto a mayor variabilidad -, no podríamos aseverar que el aumento de la prevalencia de TPNE y los cambios en las razones de prevalencias obedezcan estrictamente a la crisis económica. Probarlo requeriría un análisis causal, en el que tendría que controlarse la posibilidad de que algunas personas hayan perdido su empleo posteriormente a la aparición de la TPNE, lo que rebasa la capacidad y objetivo de este artículo.

\section{Colaboradores}

M Caicedo y E van Gameren han contribuido y colaborado en todas las etapas de trabajo. Los autores han leído y aprobado el manuscrito.

\section{Agradecimentos}

Agradecemos a la Dra. Liliana Giraldo sus valiosos comentarios al artículo. 


\section{Referências}

1. Jahoda M. Empleo y desempleo. Un análisis socio-psicológico. Madrid: Morata; 1987.

2. Micklewright J, Pearson M, Smith S. Unemployment and Early School Leaving. Economic Journal 1990; 100(400):163-169.

3. Sen A. Desigualdad y desempleo en la Europa contemporánea. Rev Internacional del Trabajo 1997; 116(2):169-187.

4. Tokman V. Desempleo juvenil en el Cono Sur: causas, consecuencias y políticas. Serie Prosur [Internet] 2003. [consultado 2011 ene 23]. Disponible en: http://library. fes.de/pdf-files/bueros/vifa-latam/c03-00907.pdf

5. Horwitz AV. An overview of sociological perspectives on definitions, causes, and responses to mental health and illness. In: Scheid TL, Brown TN, editors. A Handbook for the Study of Mental Health, Social Contexts, Theories and Systems. 2a ed. Cambridge: Cambridge University Press; 2010. p. 6-19.

6. Vedder RK, Lowell G. Racial differences in unemployment in the United States, 1890-1990. Journal of Economic History 1992; 52(3):696-702.

7. Stratton LS. Racial differences in men's unemployment. Industrial and Labor Relations Review 1993; 46(3):451-463.

8. Rodríguez, E, Allen J, Frongillo E, Chandra P. Unemployment, depression, and health: a look at the African-American community. I Epidemiol Community Health 1999; (53):335-342.

9. Caicedo M. Migración, Trabajo y Desigualdad, los Inmigrantes Latinoamericanos y Caribeños en Estados Unidos. México: El Colegio de México; 2010.

10. Kochhar R, Espinoza SC, Hinze-Pifer R. After the Great Recession: Foreign Born Gain Jobs; Native Born Lose Jobs. Pew Hispanic Center [Internet] 2010. [consultado 2010 jul 12]. Disponible en: http://www.pewhispanic org/files/reports/129.pdf

11. Caicedo M. El desempleo de la población de origen mexicano en Estados Unidos. Coyuntura Demográfica. Revista sobre Procesos Demográficos en México Hoy 2013; (3):77-83.

12. Wilson $\mathrm{W}$. The truly disadvantaged revisited: a response to Hochschild and Boxill. Ethics 1991; 101(3):593-609.

13. Organización Mundial de la Salud. ¿Qué es la salud mental? [Internet] 2007. [consultado 2014 sep 29]. Disponible en: http://www.who.int/features/qa/62/es/ index.html

14. Alvaro JL. Salud mental. In: Reyes R, editor. Diccionario Crítico de Ciencias Sociales. Terminología Científico-Social. Madrid, México: Plaza y Valdés; 2009. [Internet]. [consultado 2012 nov 22]. Disponible en: http://www. ucm.es/info/eurotheo/diccionario/S/index.html

15. Bhugra D, Jones M. Migration and mental illness. Advances in Psychiatric Treatment 2001; 7:216-223.

16. Vega W, Kolody B, Valle JR. Migration and mental health: An empirical test of depression risk factors among immigrant Mexican women. International Migration Review 1987; 21(3):512-530.

17. Vega W, Rumbaut R. Ethnic minorities and mental health. Annual Review of Sociology 1991; 17:351-383.
18. Carrasquillo O, Carrasquillo AI, Shea S. Health insurance coverage of immigrants living in the United States: differences by citizenship status and country of origin. Am J Public Health 2000; 90(6):917-923.

19. Ku L, Waidmann T. How Race/Ethnicity, Immigration Status and Language Affect Health Insurance Coverage, Access to Care and Quality of Care Among the Low-Income Population. Washington: Kaiser Family Foundation; 2003.

20. Guarnaccia PJ, Martínez I, Acosta H. Mental health in the Hispanic immigrant community: an overview. In: Finlayson M, Gonzalez MJ, Gonzalez-Ramos GM, editors. Mental Health Care for New Hispanic Immigrants: Innovative Approaches in Contemporary Clinical Practice. Binghamton: The Haworth Social Work Practice Press; 2005.

21. DuBard CA, Gizlice Z. Language Spoken and Differences in Health Status, Access to Care, and Receipt of Preventive Services Among US Hispanics. Am J Public Health 2008; 98(11):2021-2028.

22. Vega WG, Buhl-Auth JW, Meinhardt K. The prevalence of depressive symptoms among Mexicans Americans and Anglos. Am J Epidemiol 1984; 120(4):592-607.

23. Alegria M, Canino G, Shrout PE, Woo M, Duan N, Vila D, Torres M, Chen C, Meng XL. Prevalence of mental illness in immigrant and non-immigrant U.S. Latino groups. National Institute of Health. Am J Psychiatry 2008; 165(3):359-369

24. Grant B, Stinson F, Hasin D, Dawson D, Chou P, Anderson K. Immigration and lifetime prevalence of DSMIV psychiatric disorders among Mexican Americans and Non-Hispanic whites in the United States, Results from the National Epidemiologic Survey on alcohol and related conditions. Arch Gen Psychiatry 2004; 61(12):1226-1233.

25. Jahoda M, Lazarsfeld PF, Zeisel H. Marienthal. The Sociography of an Unemployed Community. Chicago: Aldine; 1971.

26. Eisenberg P, Lazarsfeld PF. The psychological effects of unemployment. Psychological Bulletin 1938; 35(6):358390

27. Linn MW. Sandifer R. Stein S. Effects of unemployment on mental and physical health. American J Public Health 1985; 75(5):502-506

28. Björklund A. Unemployment and mental health: some evidence from panel data. The Journal of Human Resources $1985 ; 20(4): 469-483$.

29. Searight HR, Handal PJ, McCauliffe TM. The relationship between public mental health admission rates, institutional constraints, and unemployment. Administration and Policy in Mental Health and Mental Health Services Research 1989; 17(1):33-42.

30. Jin RL, Shah CP, Svoboda TJ. The impact of unemployment on health: a review of the evidence. Can Med Assoc J 1995; 153(5):529-540.

31. Galić Z, Šverko B. Effects of prolonged unemployment and reemployment on psychological and physical health. Review of Psychology 2008; 15(1-2):3-10. 
32. Muller J, Delahaye B, Winocur S, Hicks R. The psychological impacts oflong-term unemployment, sex differences and activity: a case study analysis. Journal of Applied Social Behaviour 1996; 3(1):30-43.

33. Murphy GC, Athanasou J. The effect of unemployment on mental health. Journal of Occupational and Organizational Psychology 1999; 72(1):83-99.

34. Lewis G, Sloggett A. Suicide, Deprivation, and Unemployment: Record Linkage Study. BMJ 1998; 317(7168):1283-1286.

35. Kennedy S, McDonald JT. Immigrant Mental Health and Unemployment. The Economic Record 2006; 82(259):445-459.

36. Mossakowski KN. The influence of past unemployment duration on symptoms of depression among Young women and men in the United States. Am J Public Health 2009; 99(10):1826-1832.

37. Tefft N. Insights on unemployment, unemployment insurance, and mental health. J Health Econ 2011; 30(2):258-264.

38. Lennon MC, Limonic L. Work and unemployment as stressors. In: Scheid TL, Brown TN, editors. A Handbook for the Study of Mental Health, Social Contexts, Theories and Systems. 2a ed. Cambridge: Cambridge University Press; 2010. p. 213-225.

39. Garrido A. Desempleo. In: Reyes R, editor. Diccionario Crítico de Ciencias Sociales. Terminología Científico-Social. [Internet]. Madrid-México: Plaza y Valdés; 2009. [consultado 2010 mayo30]. Disponible en: http://www. ucm.es/info/eurotheo/diccionario/D/desempleo.htm

40. Del Pozo Iribarría JA, Ruiz MA, Pardo A, San Martín R. Efectos de la duración del desempleo entre los desempleados. Psicothema 2002; 14(2):440-443.

41. Schaufeli WB. Unemployment and mental health in well - and poorly - educated school leavers-leavers. In: Verhaar CHA, Jansma, LG, editors. On the Mysteries of Unemployment: Causes, Consequences and Policies. Berlin: Springer; 1992. p. 253-271.

42. Ezzy D. Unemployment and mental health: a critical review. Soc Sci Med 1993; 37(1):41-52.

43. Thoits PA. Sociological Approaches to Mental Illness. In: Scheid TL, Brown TN, editors. A Handbook for the Study of Mental Health, Social Contexts, Theories and Systems. $2^{\text {a }}$ ed. Cambridge: Cambridge University Press; 2010. p. 106-124.

44. Achótegui J. Emigrar en situación extrema: el Síndrome del inmigrante con estrés crónico y múltiple (Síndrome de Ulises). Norte de la Salud Mental 2004; 5(21):39-52.

45. Portes A, Rumbaut R. Immigrant America. A Portrait. Berkeley: University of California Press; 1994.

46. Krieger N. Embodying inequality: A review of concepts, measures and methods for studying health consequences of discrimination. Int J Health Serv 1999: 29(2):295-352.

47. Williams DR, Acosta M, Leavell JP. Race and mental health: patterns and challenges. In: Scheid TL, Brown TN, editors. A Handbook for the Study of Mental Health, Social Contexts, Theories and Systems. $2^{\text {a }}$ ed. Cambridge: Cambridge University Press; 2010. p. 268-290.
48. Kessler RC, Mickelson KD, Williams DR. The prevalence, distribution and mental health correlates of perceived discrimination in the United States. J Health Soc Behav 1999: 40(3):208-230.

49. Darity W. Employment discrimination, segregation and health. Am J Public Health 2003; 93(2):226-231.

50. Pavalko EK, Mossakowski KN, Hamilton VJ. Does perceived discrimination affect Health? Longitudinal relationships between work discrimination and women's physical and educational health. J Health Soc Behav 2003; 43(1):18-33.

51. Craig TKJ. Psychosis, migration and ethnic minority status: a story of inequality, rejection and discrimination. In: Bhugra D, Gupta S, editors. Migration and Mental Health. Cambridge: Cambridge University Press; 2011. p. 107-116.

52. Thapa SB, Hauff E. Gender differences in factors associated with psychological distress among immigrants from low- and middle-income countries-findings from the Oslo Health Study. Soc Psychiatry Psychiatr Epidemiol 2005; 40(1):78-84.

53. National Health Interview Survey Methods. [Internet]. [consultado 2014 sep 22]. Disponible en: http:// www.cdc.gov/nchs/nhis/methods.htm

54. National Health Interview Survey. Questionnaires, Datasets, and Related Documentation. [Internet]. [consultado 2014 sep 17]. Disponible en: http://www. cdc.gov/nchs/nhis/nhis_questionnaires.htm

55. Minnesota Population Center, State Health Access Data Assistance Center. Integrated Health Interview Series: Version 5.0. Minneapolis: University of Minnesota. 2012. [consultado 2010 jul 12]. Disponible en: http:// www.ihis.us

56. Kessler RC, Mroczek DK. Final version of our non-specific Psychological Distress Scale. Ann Arbor: University of Michigan; 1994.

57. Kessler RC, Andrews G, Colpe LJ, Hiripi E, Mroczek DK, Normand SLT, Walters EE, Zaslavsky AM. Short screening scales to monitor population prevalence and trends in non-specific psychological distress. Psychol Med 2002; 32(6):959-976.

58. Kessler RC, Barker PR, Colpe LJ, Epstein JF, Gfroerer JC, Hiripi E, Howes MJ, Normand SL, Manderscheid RW, Walters EE, Zaslavsky AM. Screening for Serious Mental Illness in the General Population. Arch Gen Psychiatry 2003; 60(2):184-189.

59. Schiaffino A, Rodríguez M, Pasarín MI, Regidor E, Borrell C, Fernández E. ¡Odds ratio o razón de proporciones? Su utilización en estudios transversales. Gac Sanit 2003; 17(1):70-74.

60. Bureau of Labor Statistics Labor Force Statistics from the Current Population Survey. [Internet] [consultado 2011 jul 18]. Disponible en: http://www.bls.gov/cps/ cps_over.htm

61. Markides KS, Coreil J. The health of Hispanics in the Southwestern: an epidemiologic paradox. Public Health Reports 1986; 101(3):253-265. 
62. Thomson EF, Nuru-Jeter A, Richardson D, Raza F, Minkler M. The Hispanic Paradox and Older Adults' Disabilities: Is There a Healthy Migrant Effect? Int J Environ Res Public Health 2013; 10(5):1786-1814.

63. Crimmins EM, Soldo BJ Kim JK, Alley DE. Using anthropometric indicators for Mexicans in the United States and Mexico to understand the selection of migrants and the "Hispanic paradox". Soc Biol 2005; 52(34):164-177.

64. Stompe T, Holzer D, Friedmann A, Bhugra D. Pre-migration, personality and precipitating factors. In: Bhugra D, Gupta S, editors. Migration and Mental Health. Cambridge: Cambridge University Press; 2011. p. 6878.

65. Department of Labor. Panorama de la Fuerza Laboral Latina, 5 de abril de 2012. [Internet]. [consultado 2012 nov 16]. Disponible en:

http://www.dol.gov/_sec/media/reports/HispanicLaborForce/HispanicLaborForceSpanish.pdf

Artigo apresentado em 01/07/2014

Aprovado em 21/07/2015

Versão final apresentada em 23/07/2015 